\title{
The effect of concomitant cholecystectomy and sleeve gastrectomy on morbidity in high-risk obese patients with symptomatic gallstones
}

\author{
Mürşit Dincer ${ }^{1}$, Fadli Dogan ${ }^{2}$ \\ ${ }^{1}$ Department of General Surgery, Firat University, Elazig, Turkey \\ ${ }^{2}$ Department of General Surgery, Elazig Medical Park Hospital, Elazig, Turkey
}

Videosurgery Miniinv 2019; 14 (2): 237-241

DOI: https://doi.org/10.5114/wiitm.2019.81686

\begin{abstract}
Introduction: Obesity is an independent risk factor for gallstones. In obese patients, gallstone is more symptomatic than in non-obese people.

Aim: To present the early results of laparoscopic sleeve gastrectomy (LSG) and concomitant cholecystectomy (CC) performed in patients with symptomatic gallstone accompanied by at least one additional systemic disease to obesity and to investigate its effect on morbidity.

Material and methods: Patients undergoing sleeve gastrectomy for morbid obesity between January 2016 and August 2018 were retrospectively studied. Twenty-seven patients who underwent laparoscopic sleeve gastrectomy and concomitant cholecystectomy due to symptomatic gallstone stones were included in this study. The patients were divided into two groups according to the applied surgical technique: laparoscopic sleeve gastrectomy and concomitant cholecystectomy $(n=27)$ and laparoscopic sleeve gastrectomy $(n=70)$. The results of an additional operation on these patients and their perioperative complications were evaluated.

Results: A total of 97 patients were included in the study. The mean age of the patients was $40.58 \pm 10.36$ years. There was no statistically significant difference between groups in terms of complications $(p=0.669)$. The difference in the duration of the operation was statistically significant $(p<0.001)$.

Conclusions: Concomitant cholecystectomy may be presented as an alternative surgical procedure due to the demonstration that concomitant cholecystectomy can be performed safely in comorbid obese patients, with a risk of becoming symptomatic in the rest of life at a certain rate, and low risk of complications in asymptomatic patients.
\end{abstract}

Key words: obesity, sleeve gastrectomy, gallstones, concomitant cholecystectomy, high-risk patient.

\section{Introduction}

Nowadays obesity is one of the most important health problems [1]. Morbid obesity is defined as when the body mass index exceeds $40 \mathrm{~kg} / \mathrm{m}^{2}$. The risk of comorbidities such as chronic obstructive pulmonary disease (COPD) and diabetes mellitus (DM) is higher in obese patients. Obesity is also an independent risk factor for gallstones $[2,3]$. In obese patients, gallstone is more symptomatic than in non-obese people [4]. Bariatric procedures have been accelerating with simultaneous cholecystectomy applications nowadays [5, 6]. However, the routine application of prophylactic cholecystectomy in patients with obesity surgery with gallstone has been discussed in the literature [6]. Prophylactic cholecystectomy has come onto the agenda due to 
adhesions that may occur after past surgery, but currently it is not routinely accepted [7]. Cholecystectomy is recommended in symptomatic patients $[8,9]$.

\section{Aim}

The aims of this study were to present the early results of laparoscopic sleeve gastrectomy (LSG) and concomitant cholecystectomy (CC) performed in patients with high-risk and symptomatic gallstone accompanied by at least one additional systemic disease to obesity and to investigate its effect on morbidity. Another aim of this study was to question the feasibility of prophylactic cholecystectomy in asymptomatic obese patients based on the results of this study.

\section{Material and methods}

Patients undergoing sleeve gastrectomy for morbid obesity between January 2016 and August 2018 were retrospectively studied. Twenty-seven patients who underwent laparoscopic sleeve gastrectomy and concomitant cholecystectomy due to symptomatic gallstones were included in the study in addition to obesity due to a high anesthesia score in patients with additional systemic disease such as diabetes and COPD.

Patients with previous cholecystitis, pancreatitis history, or at least one biliary colic admitted to the hospital as symptomatic gallbladder were accepted. Patients who had no systemic disease other than obesity and who had asymptomatic gallstones were excluded from the study. The short-term results of sleeve gastrectomy and simultaneous laparoscopic cholecystectomy were compared with those of 70 patients who had similar additional diseases at the same time and who underwent only laparoscopic sleeve gastrectomy. The results of an additional operation on these patients and their perioperative complications were evaluated.

\section{Statistical analysis}

In statistical analyses, the Jamovi project (2018) computer software (Jamovi Version 0.9.1.11) (retrieved from https://www.jamovi.org) (open source) program was used. Firstly, the Shapiro-Wilk test was applied to all variables to determine whether the distributions of the variables are normally distributed. Continuous data were expressed as mean \pm standard deviation or median (IQR), while categorical data were summarized as number and percentage.

The Mann-Whitney $U$ test was used to compare groups which were not normally distributed, with respect to variables and the independent samples $t$-test was used when comparing the groups in normally distributed variables. The chi-square $\left(\chi^{2}\right)$ test or Fisher's exact test was used to assess two independent categorical groups for intergroup comparisons. The level of significance was accepted as $p<0.05$.

\section{Results}

A total of 97 patients were included in the study, $62(63.9 \%)$ were female and 35 (36.1\%) were male. The mean age of the patients was $40.58 \pm 10.36$ years (range: $18-69$ years). The patients were divided into two groups according to the applied surgical technique: laparoscopic sleeve gastrectomy + concomitant cholecystectomy (LSG + CC) $(n=27)$ and laparoscopic sleeve gastrectomy (LSG) $(n=70)$. All the patients who were included in the study had antibiotic prophylaxis and deep vein thrombus prophylaxis. All patients had drainage and had a stomach tube calibrated with $37 \mathrm{Fr}$. The scores of the patients were III and IV according to American Society of Anesthesiologists (ASA) scores. There was no statistically significant difference between the two groups in terms of ASA scores.

Bleeding developed in 1 (3.7\%) patient in the LSG + CC group and 6 (8.57\%) in the LSG group while no post-operative leakage developed in any of the study patients. There was no statistically significant difference between groups in terms of complications $(p=0.669)$. The mean age of the patients in the LSG $+C C$ group was $40.7 \pm 8.2$ years and the mean age of the patients in the LSG group was $40.5 \pm 11.1$ years. There was no statistically significant difference between the groups in terms of age $(p=0.913)$. There was a statistically significant difference between the groups ( $p=0.025, p=0.003$ and $p<0.001$, respectively) when the groups were compared according to gender, body mass index (BMI) and surgery duration. The majority of patients in the LSG + CC group were female (81.48\%). The surgery duration of the LSG + CC group was longer ( $65.7 \pm 8.5 \mathrm{~min}$ vs. $57.1 \pm 8.7 \mathrm{~min}$ ) and patients in the LSG group had a higher BMI (46.83 vs. 42.9).

When participants were questioned for additional diseases, there was a statistically significant 
difference between groups in terms of presence of hypertension and DM $(p<0.001)$. Diabetes mellitus (95.71\%) in the LSG group and HT $(22.72 \%)$ in the LSG + CC group were frequent. There was no statistically significant difference between the groups when blood glucose level, glycated hemoglobin $\left(\mathrm{HbA}_{1 \mathrm{c}}\right)$ level, transfusion need during surgery and duration of hospital stay were evaluated before the operation $(p>0.05)$. Comparisons according to demographic and clinical characteristics of the patients are shown in Table I.

\section{Discussion}

In patients with morbid obese, gallstones are seen at the rate of $19-45 \%[4,10]$. Gallstones are more common in women than in men, independently of age [11]. In our study, 62 (63.9\%) patients were female. Cholelithiasis is asymptomatic in some of these patients. Whether it is necessary to perform cholecystectomy in morbidly obese patients with asymptomatic cholelithiasis has been discussed in the literature [7-10, 12]. Sleeve gastrectomy is now often used alone as a restrictive bariatric procedure $[4,13,14]$. The approach to be applied in the presence of cholelithiasis is also unclear in patients who are to undergo sleeve gastrectomy [7, 15]. It has been reported in the literature that concomitant prophylactic cholecystectomy during bariatric surgery prolongs the surgical and hospital stay and increases morbidity $[7,16]$. Studies suggesting prophylactic cholecystectomy due to adhesions that may occur after previous surgery are also available in the literature. Conversely, a meta-analysis conducted by Warschkow et al. showed that the complication rate of cholecystectomy after bariatric surgery was low and emphasized that the prophylactic cholecystectomy should not be routinely performed [17]. In our current approach we do not recommend prophylactic cholecystectomy as an absolute indication during sleeve gastrectomy. However, in the studies of Raziel et al. and Sioka et al., the group of patients who required post-sleeve gastrectomy cholecystectomy was reported to have a frequency of $9.3 \%$ and $13 \%$, respectively $[4,18]$

In this study, there was no significant difference in complications and duration of hospital stay between the laparoscopic cholecystectomy group and the other groups. Based on these findings, the question "Can laparoscopic cholecystectomy be offered concurrently in terms of future risks in cases with asymptomatic cholelithiasis?" arose. We think that it is important to share this issue with patients and to consider the preference of the patient.

It has been reported in the literature that in cases of simultaneous cholecystectomy with obesity surgery extended the surgical duration by 18-49 min $[10,12]$. In our study this value was found to be about $8.6 \mathrm{~min}$.

There are reports that concomitant cholecystectomy in patients undergoing bariatric surgery prolongs hospital stay and increases morbidity $[19,20]$. This study was conducted on a group of patients with higher risk than those having obesity without additional disease. Despite all these factors, there was no significant difference in the duration of stay in hospital and morbidity between the two groups in this study.

While planning this study, simultaneous cholecystectomy was more difficult to implement than standard cholecystectomy in patients with morbid obesity who undergo sleeve gastrectomy due to fatty liver, standard cholecystectomy trocar entry sites not being used. As a result of this difficulty, the duration of surgery was prolonged and it was predicted that there would be an increase in morbidity due to the duration of prolonged anesthesia. However, no statistically significant difference was found between the two groups in terms of morbidity regardless of surgery duration.

Cholecystectomy in obese patient may be difficult not only due to obesity but also due to comorbidities such as pulmonary and vascular problems. When it is performed after important weight loss it may actually be easier. On the other hand, the approach suggested by this study eliminates the need for second operations and second anesthesia, which may be beneficial for the patient

The present study had some limitations. The limited number of patients was the most important one. The intervention group is small. Single-center experience was another limitation for a reliable evaluation. Also, the patients were not equally distributed between the groups. However, the presented data mostly concerned the periprocedural experience and this study was performed with a higher risk and symptomatic gallstone accompanied by at least one additional systemic disease besides obesity. Efforts were made to ensure homogenization between the groups for high risk. This was not a study that reported the results of a prophy- 
Table I. Comparison of demographic and clinical characteristics of patients

\begin{tabular}{|c|c|c|c|c|}
\hline \multirow{2}{*}{\multicolumn{2}{|c|}{ Variables }} & \multicolumn{2}{|c|}{ Surgery } & \multirow[t]{2}{*}{$P$-value } \\
\hline & & $\mathrm{LSG}+\mathrm{CC}(n=27)$ & LSG $(n=70)$ & \\
\hline \multicolumn{2}{|l|}{ Age [years] } & $40.7 \pm 8.2$ & $40.5 \pm 11.1$ & $0.913^{1}$ \\
\hline \multicolumn{2}{|l|}{ BMI [kg/m²] } & $42.9(40.8-47.5)$ & $46.8(44.7-49.2)$ & $0.003^{2}$ \\
\hline \multirow[t]{2}{*}{ Gender } & Male & $5(18.52)$ & $30(42.86)$ & $0.025^{3}$ \\
\hline & Female & $22(81.48)$ & $40(57.14)$ & \\
\hline \multirow[t]{2}{*}{ Tissue adhesive } & Yes & $22(81.48)$ & $70(100)$ & $0.001^{4}$ \\
\hline & No & $5(18.52)$ & $0(0)$ & \\
\hline Leakage test & No & $27(100)$ & $70(100)$ & - \\
\hline \multirow[t]{2}{*}{ Complication } & Bleeding & $1(3.7)$ & $6(8.57)$ & $0.669^{4}$ \\
\hline & No & $26(96.3)$ & $64(91.43)$ & \\
\hline Drain & Yes & $27(100)$ & $70(100)$ & - \\
\hline Antibiotic & Yes & $27(100)$ & $70(100)$ & - \\
\hline DVT prophylaxis & Yes & $27(100)$ & $70(100)$ & - \\
\hline \multicolumn{2}{|l|}{$\mathrm{HbA}_{1 c}$} & $6.2(6.0-6.9)$ & $6.4(6.1-7.4)$ & $0.284^{2}$ \\
\hline \multicolumn{2}{|l|}{ Glucose } & 125 (107-140) & 117 (102-183) & $0.423^{2}$ \\
\hline \multicolumn{2}{|c|}{ Duration of stay in hospital [days] } & $4(3-4)$ & $3(3-4)$ & $0.131^{2}$ \\
\hline \multicolumn{5}{|l|}{ Additional disease: } \\
\hline \multirow[t]{2}{*}{ Asthma } & Yes & $1(3.7)$ & $0(0)$ & $0.278^{4}$ \\
\hline & No & $26(96.3)$ & $70(100)$ & \\
\hline \multirow[t]{2}{*}{ DM } & Yes & $15(55.56)$ & $67(95.71)$ & $<0.001^{4}$ \\
\hline & No & $12(44.44)$ & $3(4.29)$ & \\
\hline \multirow[t]{2}{*}{ HT } & Yes & $6(22.22)$ & $0(0)$ & $<0.001^{4}$ \\
\hline & No & $21(77.78)$ & $70(100)$ & \\
\hline \multirow[t]{2}{*}{ Epistaxis } & Yes & $0(0)$ & $1(1.43)$ & $0.999^{4}$ \\
\hline & No & $27(100)$ & $69(98.57)$ & \\
\hline \multirow[t]{2}{*}{ DVT } & Yes & $1(3.7)$ & $0(0)$ & $0.278^{4}$ \\
\hline & No & $26(96.3)$ & $70(100)$ & \\
\hline \multirow[t]{2}{*}{ GERD } & Yes & $3(11.11)$ & $20(28.57)$ & $0.070^{3}$ \\
\hline & No & $24(88.89)$ & $50(71.43)$ & \\
\hline \multirow[t]{2}{*}{ CAD } & Yes & $1(3.7)$ & $0(0)$ & $0.278^{4}$ \\
\hline & No & $26(96.3)$ & $70(100)$ & \\
\hline \multirow[t]{2}{*}{ COPD } & Yes & $2(7.41)$ & $1(1.43)$ & $0.186^{4}$ \\
\hline & No & $25(92.59)$ & $69(98.57)$ & \\
\hline \multirow[t]{2}{*}{ OSAS } & Yes & $1(3.7)$ & $0(0)$ & $0.278^{4}$ \\
\hline & No & $26(96.3)$ & $70(100)$ & \\
\hline \multirow[t]{3}{*}{ Transfusion need: } & $\begin{array}{l}\text { Fresh frozen } \\
\text { plasma }\end{array}$ & $2(2-2)$ & $1(1-4)$ & $0.816^{2}$ \\
\hline & $\begin{array}{l}\text { Erythrocyte } \\
\text { suspension }\end{array}$ & $2(2-2)$ & $4(1-4)$ & $0.763^{2}$ \\
\hline & Full blood & - & $1(1-1)$ & - \\
\hline \multicolumn{2}{|l|}{ Calibration - $37 \mathrm{Fr}$} & $27(100)$ & $70(100)$ & - \\
\hline \multicolumn{2}{|c|}{ Surgery duration [min] } & $65.7 \pm 8.5$ & $57.1 \pm 8.7$ & $<0.001^{1}$ \\
\hline
\end{tabular}

LSG - laparoscopic sleeve gastrectomy, CC - concomitant cholecystectomy, DVT - deep vein thrombosis, HT - hypertension, DM - diabetes mellitus, GERD gastroesophageal reflux disease, CAD - coronary artery disease, COPD - chronic obstructive pulmonary disease, OSAS - obstructive sleep apnea syndrome,

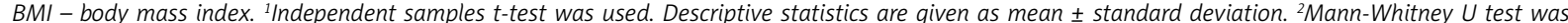
used. Descriptive statistics are given as median (IQR). ${ }^{3}$ Pearson $\chi^{2}$ test was used. Descriptive statistics are given as number (\%). ${ }^{4}$ Fisher's exact test was used. Descriptive statistics are given as number (\%). 
lactic cholecystectomy. Patients with asymptomatic cholelithiasis and patients with symptomatic gallstones without additional risk factors other than obesity were excluded. One of the aims of this study was to question the feasibility of prophylactic cholecystectomy in asymptomatic obese patients. For all these reasons, we think it will contribute to the literature. Although there is no similar study in literature, there is a need for prospective studies with more patients in order to evaluate the results more closely.

\section{Conclusions}

Concomitant cholecystectomy in morbidly obese patients with comorbid diseases and symptomatic gallstones has not been shown to increase morbidity. The LSG and CC is a safe and effective method in high-risk morbidly obese patients. As a result of this study, concomitant cholecystectomy may be presented as an alternative surgical procedure due to the demonstration that concomitant cholecystectomy can be performed safely in comorbid obese patients, with a risk of becoming symptomatic in the rest of life at a certain rate, and low risk of complications in asymptomatic patients.

\section{Conflict of interest}

The authors declare no conflict of interest.

\section{References}

1. Kang JH, Le QA. Effectiveness of bariatric surgical procedures. A systematic review and network meta-analysis of randomized controlled trials. Medicine 2017; 96: 46 (e8632).

2. Ligibel JA, Alfano CM, Courneya KS, et al. American Society of Clinical Oncology position statement on obesity and cancer. J Clin Oncol 2014; 32: 3568-74.

3. Dakour-Aridi HN, El-Rayess HM, Abou-Abbas H, et al. Safety of concomitant cholecystectomy at the time of laparoscopic sleeve gastrectomy: analysis of the American College of Surgeons National Surgical Quality Improvement Program database. Surg Obes Rel Dis 2017; 13: 934-42.

4. Raziel A, Sakran N, Szold A, Goitein D. Concomitant cholecystectomy during laparoscopic sleeve gastrectomy. Surg Endosc 2015; 29: 2789-93.

5. Kohn GP, Galanko JA, Overby DW, Farrell TM. Recent trends in bariatric surgery case volume in the United States. Surgery 2009; 146: 375-80.

6. Mason EE, Renquist KE. Gallbladder management in obesity surgery. Obes Surg 2002; 12: 222-9.

7. Lee JH, Han G, Kim YJ, et al. A technique for simultaneous cholecystectomy during bariatric surgery. JSLS 2015; 19: e2015.00072.
8. Quesada BM, Kohan G, Roff HE, et al. Management of gallstones and gallbladder disease in patients undergoing gastric bypass. World I Gastroenterol 2010; 16: 2075-9.

9. Yardimci S, Coskun M, Demircioglu S, et al. Is concomitant cholecystectomy necessary for asymptomatic cholelithiasis during laparoscopic sleeve gastrectomy? Obes Surg 2018; 28: 469-73.

10. Coşkun H, Hasbahçeci M, Bozkurt S, et al. Is concomitant cholecystectomy with laparoscopic sleeve gastrectomy safe? Turk J Gastroenterol 2014; 25: 624-7.

11. Shaffer EA. Gallstone disease: epidemiology of gallbladder stone disease. Best Pract Res Clin Gastroenterol 2006; 20: 981-96.

12. Tucker ON, Fajnwaks P, Szomstein S, Rosenthal RJ. Is concomitant cholecystectomy necessary in obese patients undergoing laparoscopic gastric bypass surgery? Surg Endosc 2008; 22: 2450-4.

13. Almogy G, Crookes PF, Anthone GJ. Longitudinal gastrectomy as a treatment for the high-risk super-obese patient. Obes Surg 2004; 14: 492-7.

14. Melissas J, Koukouraki S, Askoxylakis J, et al. Sleeve gastrectomy: a restrictive procedure? Obes Surg 2007; 17: 57-62.

15. Li VK, Pulido N, Martinez-Suartez P, et al. Symptomatic gallstones after sleeve gastrectomy. Surg Endosc 2009; 23: 2488-92.

16. Tsirline VB, Keilani ZM, El Djouzi S, et al. How frequently and when do patients undergo cholecystectomy after bariatric surgery? Surg Obes Relat Dis 2014; 10: 313-21.

17. Warschkow R, Tarantino I, Ukegjini K, et al. Concomitant cholecystectomy during laparoscopic Roux-en- $Y$ gastric bypass in obese patients is not justified: a meta-analysis. Obes Surg 2013; 23: 397-407.

18. Sioka E, Zacharoulis D, Zachari E, et al. Complicated gallstones after laparoscopic sleeve gastrectomy. J Obes 2014; 2014: 468203.

19. Hamad GG, Ikramuddin S, Gourash WF, Schauer PR. Elective cholecystectomy during laparoscopic Roux-en-Y gastric bypass: is it worth the wait? Obes Surg 2003; 13: 76-81.

20. Worni M, Guller U, Shah A, et al. Cholecystectomy concomitant with laparoscopic gastric bypass: a trend analysis of the nationwide inpatient sample from 2001 to 2008. Obes Surg 2012; 22: $220-9$

Received: 25.11.2018, accepted: 8.01.2019. 